\section{TARGETED DELIVERY OF A PD-L1-BLOCKING SCFV BY CAR-NK CELLS SHOWS POTENTIAL AS A NEW APPROACH TO IMMUNOTHERAPY FOR GLIOBLASTOMA}

Jiao Wang*, Sandro Matosevic. Purdue University, West Lafayette, IN, USA

Background Despite advances in treatment, glioblastoma (GBM) remains an incurable primary brain tumor with a median survival of only 15 months, highlighting the need for new therapeutic approaches. Natural killer (NK) cells, innate cytotoxic effectors, are showing potential for cancer immunotherapy including GBM. Even though GBM tumors are infiltrated by NK cells. ${ }^{1}$ However, their antitumor activities are impaired by the immunosuppressive tumor microenvironment (TME) via various mechanisms, including adenosine-mediated downregulation of NKG2D. ${ }^{2}$ Therefore, it is critical to understand more about how the TME in GBM modulates the NK cell-mediated immunity so that we can develop novel NK cellbased therapies specifically for GBM.

Methods We isolated human peripheral blood NK cells from healthy volunteer donors. U87MG and GBM43 were used as GBM targets. We co-cultured NK cells with GBM cells and measured the PD-L1 expression on the NK cells. We built a transwell co-culture system to evaluate the crosstalk between NK and GBM cells on the PD-L1 expression on GBM target cells. We then generated gene-modified NK cells expressing an NKG2D.CAR which secretes anti-PD-L1 scFv locally in the TME. These NK cells are being evaluated for their efficacy against GBM both in vitro and in vivo.
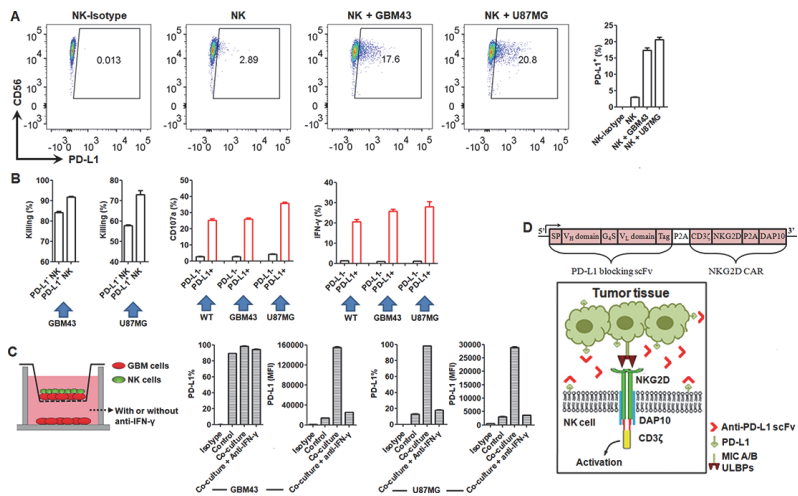

Abstract 150 Figure 1 (A) PD-L1 expression on human primary NK cells after co-incubation with different types of cancer target cells, including U87MG (human glioblastoma cell line) and GBM43 (a human patient-derived glioblastoma cell) cells at a $E / T$ ratio of 10 for $24 \mathrm{~h}$, respectively; (B) In vitro anti-GBM activities of PD-L1- NK cells vs PD-L1 + NK cells, including killing, degranulation (CD107a), and IFN- $\gamma$ secretion; (C) Setup of transwell system for evaluation of PD-L1 expression on GBM target cells upon co-culture with NK cells; (E) Multifunctional genetically-engineered NK cells for GBM immunotherapy. Data are presented as the mean \pm SEM

Results We have found that GBM target cells can upregulate PD-L1 expression on NK cells (figure 1A). And induced PD$\mathrm{L} 1+\mathrm{NK}$ cells present better in vitro anti-GBM activity, including higher killing, degranulation and IFN- $\gamma$ release (figure $1 \mathrm{~B})$. We have revealed that the expression of PD-L1 on GBM cells gets further boosted after crosstalk with NK cells, which is dependent on the induced IFN- $\gamma$ release (figure 1C). Accordingly, we have designed and synthesized a multifunctional CAR construct that enables NK cells to express a secretable PD-L1-blocking $\mathrm{scFv}$ and a CAR redirected against ligands for NKG2D (figure 1D).

Conclusions After direct contact with GBM cells, the increased population of PD-L1+ NK cells with superior cytolytic functions have been found for the first time. Also, NK cells cocultured with GBM cells can further trigger PD-L1 upregulation on GBM cells. Stimulated by these results, we have been generating $\mathrm{NK}$ cells that can, at once, secrete the PD-L1blocking scFv to target PD-L1 both expressed on $\mathrm{NK}$ and GBM cells, and express the NKG2D.CAR to specifically target its ligands on GBM cells. We are currently testing the in vitro and in vivo therapeutic efficacy of these engineered NK cells, which we believe could be used as a promsing immunotherapy for GBM.

\section{REFERENCES}

1. Wang J, Matosevic S. NT5E/CD73 as correlative factor of patient survival and natural killer cell infiltration in glioblastoma. J Clin Med 2019;8(10):1526.

2. Wang J, Lupo KB, Chambers AM, Matosevic S. Purinergic targeting enhances immunotherapy of $\mathrm{CD} 73+$ solid tumors with piggyBac-engineered chimeric antigen receptor natural killer cells. J Immunother Cancer 2018;6(1):136.

http://dx.doi.org/10.1136/jitc-2021-SITC2021.150 